\title{
Platform Models for Sustainable Internet Regulation
}

\author{
kc claffy and David D. Clark \\ kc@caida.org and ddc@csail.mit.edu
}

August 15, 2013

\begin{abstract}
The dynamic nature of the telecommunications industry, with its rapidly changing technology and industry structure, presents a serious challenge to the theory and practice of regulation, which has a slower time scale and a tendency to embed assumptions about technology and industry into regulation. This paper proposes a model that attempts to capture two durable and persistent features of today's telecommunications ecosystem: the use of layered platforms to implement desired functionality; and interconnection between actors at different platform layers. We use platform theory, and in particular theories of multi-sided platforms (MSPs), to focus on key technical and business aspects of today's industry. We use an MSPaware layered model of the ecosystem to explore several recent and impending innovations in the ecosystem that have been naively conflated with the global Internet, illuminate their differences, and describe how regulators could use our model to more rigorously consider them. Finally, to illustrate its potential as a baseline for future research, we briefly consider how this model can help scope consistent policy discourse of three open questions: specialized services, minimum quality regulations ("the dirt road" problem), and structural separation.
\end{abstract}

\section{Introduction}

A significant challenge in developing regulatory theory to support communications policy is the highly dynamic technology and business practices in the evolving Internet. Traditional regulatory theory in the telecommunications sector relied on simple conceptions of technology, such as copper pairs carrying telephone service to homes. Innovative uses of that copper pair (e.g. DSL) and advanced technologies such as hybrid fiber-coax (HFC), fiber and wireless, have led to definitional confusion, litigation, and a dauntingly complex, poorly understood networked ecosystem. Recent trends toward convergence of virtually all communications services using the Internet Protocol (IP), both in public and private networks, render the complexity and ambiguity even worse.

The goal of this paper is to present a model of communications technology and industry practice that is general enough to survive current rates of innovation and evolution, and stable enough to support relevant regulatory theory. We draw on two fundamental elements of today's telecommunications ecosystem: the use of layered platforms to implement functionality desired by either users or providers; and interconnection between actors at different platform layers to construct a larger fabric. The concepts of platforms, layering, and interconnection are not new; but our model combines them in a way that captures both stable and dynamic aspects of the technology and business practices of the ecosystem, while abstracting away details that confuse more than clarify to regulatory debates.

Section 2 describes a taxonomy of platforms classified across two dimensions: how they are constructed (one or multiple firms) and how they are used (only by the platform owners or by 
others). The Internet and its larger ecosystem is actually many layers of platforms that span all four combinations of these two dimensions. We augment this classification with a well-accepted model of contemporary interconnection patterns among ISPs, and find that the resulting picture brings some clarity to differences among several recent Internet-related service offerings and their regulatory implications. The highly dynamic nature of the industry implies that technical details of various platform layers will change faster than regulatory debate can possibly resolve, but our two-dimensional platform matrix allow reasoning that is independent of these technical details: whether they are internal or industry platforms, and whether they are single or multi-firm platforms. Equipped with this taxonomy, we discuss two platform layers of the ecosystem that seem particularly durable: the global Internet and the single-firm IP platform. A third emerging platform innovation - the multi-firm IP platform - is likely to also become common, which raises significant challenges for regulators. Section 3 explores how a platform-aware model can usefully distinguish among services running on different IP-based platforms, as well as the possible behavior of those platform owners, in a way that offers a cleaner derivation and clearer interpretation of their regulatory implications. Our underlying premise is that regulation attached to stable layers is likely to be more durable, and Section 4 considers how the framework of platforms we have developed can help scope consistent policy and regulation of the Internet. We study three policy questions of interest in a platform context: specialized services, minimum quality regulations ("the dirt road" problem), and structural separation Section 5 offer some concluding thoughts and suggests future directions of research and debate.

\section{Platforms: their nature and layered structure in the Internet}

A platform is a technology providing a set of service capabilities on top of which many different products can be developed and deployed. Operating systems (e.g., Microsoft Windows, iOS, Android) are platforms - they support a wide range of applications and services, often provided by many third-party providers. The owner of a successful platform may acquire powerful advantages in the related industrial ecosystem. Factors that influence the ability to create and control a successful platform include the dynamics of gaining and holding market share, network externalities and control of intellectual property.

In the context of industrial structure, Gawer [12] described three classes of platform: internal, supply chain and industry. Internal platforms are developed and exploited internal to a firm to reduce cost and improve flexibility and time to market for product development. Examples of internal platforms are often found in manufacturing industries such as automotive and electronics. Supply chain platforms are produced and exploited by a set of firms, linked in collaborations and alliances. An industry platform is produced by one or a set of firms and then made available to other entities (complementors) to develop products on top of that platform.

To clarify their role in our model, Figure 1 depicts four types of platforms (and examples of each) along two dimensions: construction and use. The upper row corresponds to Gawer's industry platform, although we distinguish between those constructed by one vs. multiple firms. The right column generalizes Gawer's supply chain platform to a multi-firm platform, to include cases where firms are peers or partners. Gawer's analysis still applies to multi-firm platforms, i.e., relationships among firms producing the platform influence which firms may have sufficient market power to define and regulate the platform, or even destabilize it in the process of trying to maximize profit. 
For example, Microsoft Windows is an industry platform, defined and developed by a single firm that controls it through intellectual property licensing and other methods to limit appropriation of the platform by competitors. The Internet as we know it today is a multi-firm industry platform, constructed by multiple firms interconnecting their networked assets based on (mostly) open standards. One of the most successful industry platforms in history as measured by the range of innovative applications built by complementors using it, the Internet and its larger ecosystem is actually many layers of platforms that span all four quadrants of Figure 1.

The Web is also a multi-firm platform, implemented at a layer above the basic Internet transport capability, and applications using this higher-level web platform can themselves serve as a platforms for other application developers. The Web, like the Internet, is defined by standards, mostly set by the World Wide Web Consortium (W3C) hosted at MIT, with many actors involved in both setting the standards and implementing them in web servers and browsers. On top of the Web platform one can find further examples of platforms. Facebook is a single-firm industry platform for complementors who build on top of it. In contrast to the Web platform, which is largely characterized by open standards, the Facebook platform is dictated by its owner, shaped only by any pressures from their complementors and users that they choose to respect. While there have been fears that a powerful player might try to distort a web standard using the market share of their browser, the need for interoperability among tools (firms) seems to have prevented serious fragmentation of the Web standards. ${ }^{1}$

The repeating platform structure of the Internet also appears below the network layer, down to the physical layer of routers and fibers. Internet service providers provision routers and fiber in order to build and maintain their parts of the Internet. But these technologies also enable individual providers to construct a stack of internal platforms to support a range of services by the firm, or to create additional industry platforms for complementors. In network architecture language, these repeating layers may multiplex, i.e., simultaneously support, many higher-level services. Long distance fibers are multiplexed by using different colors of light (lambdas) to carry different data streams. These data streams are often multiplexed using a technology called Multi-Protocol Label Switching (MPLS), which breaks the data stream into packets. The most common format for these packets is the Internet Protocol (IP), which results in an IP-based platform. From the perspective of each ISP, their IP platform is a single-firm platform, while the Internet is a multi-firm platform that happens to use the same protocol. At the same time, lower layers can serve as both an internal platform and an industry platform, i.e., the owner of a fiber infrastructure can both use internally and sell to others a fiber, a lambda, a share of an MPLS service, a range of IP-based services, or access to the public Internet.

\footnotetext{
${ }^{1}$ For a range of commentary on powerful actors manipulating key web standards, see for example http://www.internetnews.com/dev-news/article.php/83051/Web+Standards+Group+Criticizes+IE+5.htm (1999) or http:/ /developers.slashdot.org/story/12/08/10/2152236/microsoft-picks-another-web-standards-fight (2012).
} 


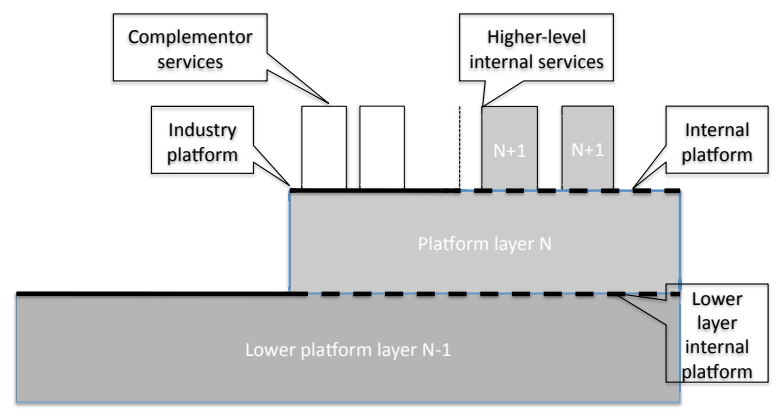

(a) Platforms sitting on lower layer platforms that can provide both an industry and internal platform to services above.

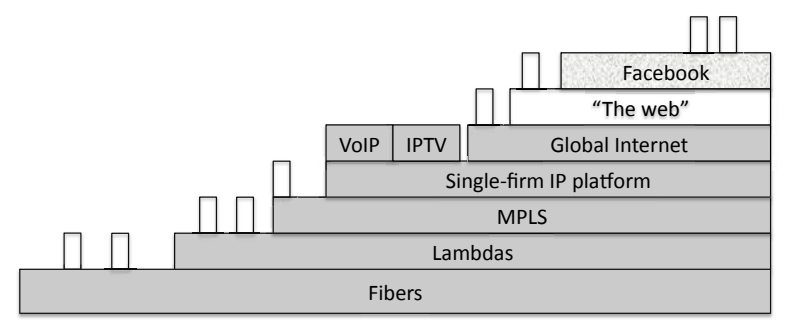

(b) In the Internet ecosystem, fiber owners may be able to create multiple platform layers, each one serving as an industry platform as well as an internal platform for the development of higher-level services.

Figure 2: Illustration of platform layers and their role in the Internet ecosystem

The significance of these layers inspire the repeating platform layer dimension of our model. Figure 2(a) illustrates how each layer sits on a lower-layer platform that supports a range of internal as well as complementor services; Figure 2(b) instantiates an example set of such platform layers in the Internet ecosystem. Layered models have been used for at least a decade to explain the structure of network technology, and to reason about regulatory treatment [19, 23, 24]. More recently, Jordan [15] argued that a critical demarcation point is between the Internet Protocol layer (and below) and those layers above the Internet layer (the application or complementor layers). He believes this delineation would not only recover some meaning to the telecommunications vs. information service terminology in the U.S. Telecommunications Act, but would promote innovation at higher layers in the industry by limiting regulation to the lower layers to prevent vertical foreclosure. Our model differs from his in that we articulate the distinction between the multi-firm industry Internet platform and the IP platform below the Internet; our goal is to avoid simplifications that mask aspects of layering that are relevant to regulatory analysis.

One of our objectives is to identify specific platform layers that are likely to be stable over time, because we argue that stable layers should be the focus of regulatory attention. The physical layer in this repeating layering has a durable character; its creation is typically capital-intensive and its evolution constrained accordingly. However, technology evolution and dynamism bring tremendous variability to the intermediate lower layers of this stack, resulting in different intermediate platforms, so any attempt to attach regulatory burdens to a specific technical implementation of a layer runs the risk of rapid irrelevance. For example, in contrast to the MPLS in Figure 2(b), the HFC infrastructure of a modern cable access system implements multiplexing using platform layers based on $6 \mathrm{Mhz}$ channel allocations and a protocol called DOCSIS. Historically contingent details of this cascade of platforms will not matter to a general and durable model. In particular, a durable model will be able to identify key features that merit different regulatory treatment of different layers, regardless of the technical details of a given layer at a given time. Such a model will be particularly helpful in the analysis of imminent, economically inevitable platform innovations about to shatter our already cracking models of communication regulation. The first such innovation we explore is the "single-firm converged IP platform", often naively conflated with the public Internet. 


\subsection{The single-firm IP platform}

Most Internet Service Providers have historically provided many services in addition to the public Internet. These services, such as telephone and cable television, create additional revenue opportunities, perhaps with higher margins than classic Internet service. Today, a common technical approach to providing these other services is for a firm to build a single-firm IP platform, a converged service layer based on the Internet protocols (IP), over which the infrastructure owner can offer its own voice (VoIP) or video (IPTV) services, as well as to provide an industry platform such as the public Internet. The use of the single-firm IP platform can support enhanced service qualities (e.g., QoS) that may not be permitted on the Internet platform, due to regulatory restrictions. The term converged captures the idea that this layer brings together disparate lower-level technologies under one service interface. The IP platform is a particularly powerful and successful service because the Internet protocols were designed to support exactly such an objective: to provide a layer that implements a uniform service interface on top of a variety of lower level technologies and platform interfaces.

This new reality changes the context for reasoning about the role and necessity of regulation. Under the assumption that the major purpose of the IP platform was to implement the public Internet, regulatory proposals that tried to constrain the relationship between the ISP and the complementors (e.g., "network neutrality" regulations) considered only the industry platform that provided the global Internet. Proponents of network neutrality (or "reasonable network management," as the FCC has termed it [8]), are concerned with the potential chilling effect on complementors if the Internet provider favored (in pricing or performance) their own higher-level services over products and services from complementors. But the regulatory proposition is more complex if the owner of the single-firm IP platform can also sell consumer-facing services on top of this internal platform instead of or as well as the global Internet.

We cannot easily dismiss these multiple uses of the single-firm IP platform, since they may be the only way to recover the costs of operating the underlying infrastructure in a competitive market. For example, access ISPs today offer what is sometimes called the triple play over their access technology voice, video and Internet; revenues from all of these services cover the costs of the infrastructure. $^{2}$

As an example of a service offered over a single-firm IP platform, Comcast recently introduced the Xfinity Xbox IPTV service,

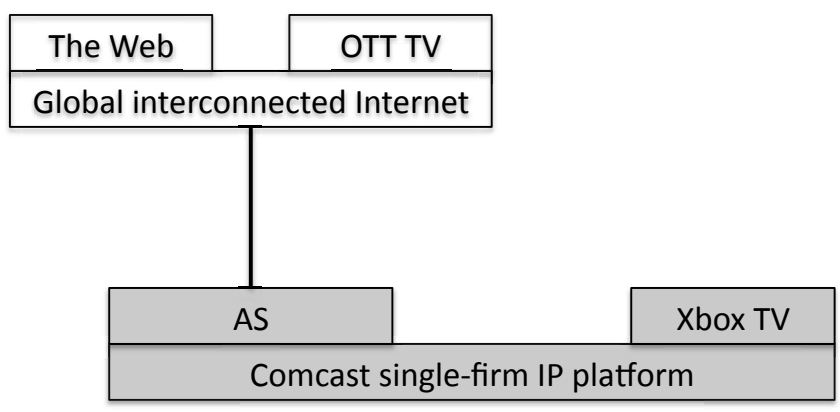

Figure 3: Delivery of Comcast Xfinity video over their converged IP platform, in contrast to over the top (OTT) video being delivered by competitors over the Internet. which carries on-demand television content over IP to a customer's Xbox for display on an attached monitor television (Figure 3) [16]. Comcast described this service as a consumer-facing service running on top of their single-firm IP platform, not the public Internet platform, and thus asserted that it was not covered by the obligations against discrimination imposed by the FCC's Open Internet Report and Order. Therefore they exempted the usage attributed to this service from the monthly quota on their Internet service. Critics argued that the Xfinity Xbox service should be viewed as operating on the global Internet platform, and therefore Comcast should (under the Open Internet Report and Order) treat it equally to other video services and include it

\footnotetext{
${ }^{2}$ The FCC concurred with this assessment of the economics of broadband access in their ruling on local franchising of cable competitors [7, para 51].
} 


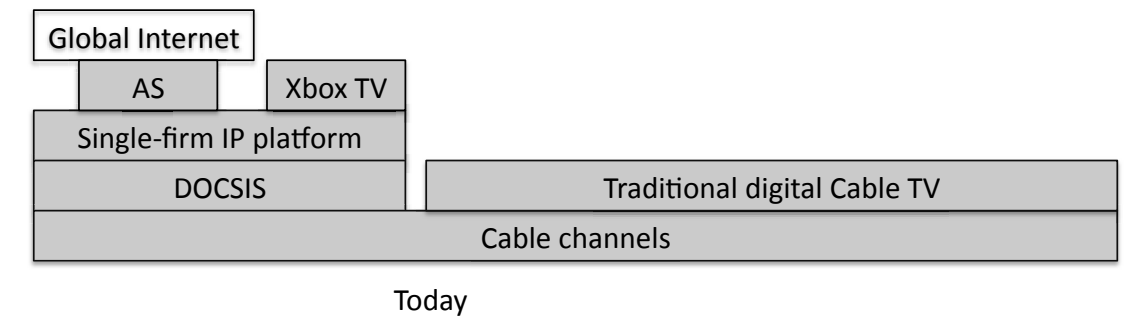

\begin{tabular}{|c|c|c|}
\hline \multicolumn{3}{|c|}{ Global Internet } \\
\hline AS & Xbox TV & IP-based TV \\
\hline \multicolumn{3}{|c|}{ Single-firm IP platform } \\
\hline \multicolumn{3}{|c|}{ DOCSIS } \\
\hline \multicolumn{3}{|c|}{ Cable channels } \\
\hline
\end{tabular}

Future

Figure 4: Most cable TV providers will migrate TV onto their single-firm converged IP platform. No IP interoperation (i.e. over a multi-firm platform) is required to deliver IPTV.

in the cap. ${ }^{3}$

This debate will intensify as all of cable television service will eventually move to this singlefirm IP platform layer model, i.e., TV will become IPTV (figure 4), which does not imply it is running as just another application on the global Internet. New services can be expected to emerge as well-services that have no prior regulatory history as cable TV and telephony do. A challenge for regulators is to assess the implications, both positive and negative, of the emergence of consumerfacing services offered by facilities owners over their single-firm (internal) IP platform.

Further complicating the analysis, a firm could convert their internal platform to an external (industry) platform, by allowing complementors access (i.e., direct interconnection) to this platform (thus moving from the lower left to the upper left quadrant of the matrix in Figure 1). This possibility reveals a fundamental question: if both the single-firm IP platform and the global Internet are supporting complementors, perhaps some of the same complementors, in what respects are they different platforms? Alternatively, two firms might agree to interconnect their internal IP platforms for a specific purpose, shifting along the other dimension of the platform matrix (from the lower left to the lower right quadrant of Figure 1).

Before we explore the implications of these two types of interconnection, we summarize the four important aspects of the ecosystem our model has captured this far. First, the ecosystem has many layers, sometimes recursive (IP on top of another IP layer), and with technical details that change rapidly enough that a stable and general model of regulation will have to ignore these technical details. Second, one can reason independent of these technical details by first characterizing platforms along the two dimensions in our matrix: whether they are internal or industry platforms, and whether they are single or multi-firm platforms. Third, the emerging single-firm IP platform is likely to be stable and persistent, which means our model has at least two durable layers: the global Internet and the single-firm IP platform. Fourth, we anticipate the emergence of a third stable platform in the IP ecosystem: a multi-firm IP platform, which may serve both as an industry or an internal platform. We next examine issues related to such multi-

\footnotetext{
${ }^{3}$ For one perspective on this debate see http://gigaom.com/2012/03/27/the-technical-and-legal-realities-ofcomcasts-xbox-cap-spat/
} 


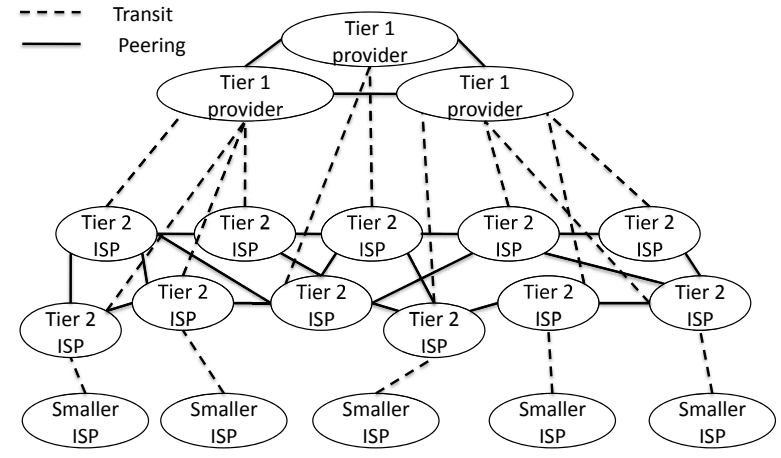

(a) Physical representation of Internet interconnection.

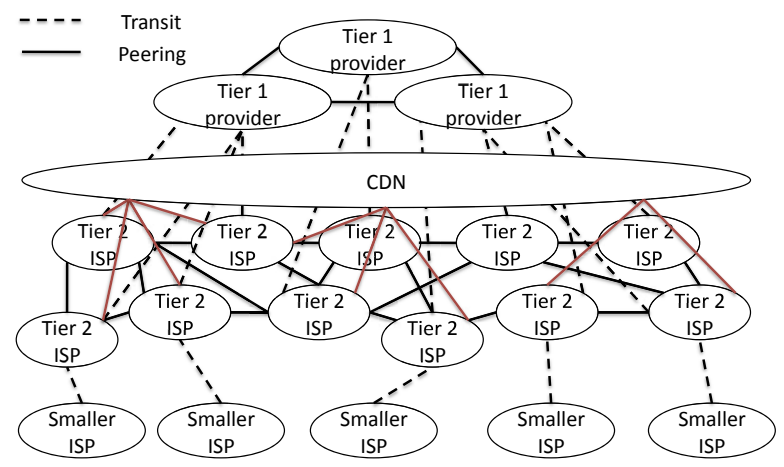

(b) The addition of a content delivery network (CDN) to the mix of interconnections.

Figure 5: Both the multi-firm production of the global Internet and the interconnection of complementors such as CDNs may manifest as physical links in a diagram of the Internet.

firm platforms, of which the Internet itself is the most recognized one.

\subsection{From single-firm platform to multi-firm platform}

We have described the global Internet as an industry platform composed of an interconnected mesh of many single-firm IP platforms. Interconnection is the process, evolving for the last several decades, of building a multi-firm platform out of the single-firm IP platform of each ISP.

Internet interconnection patterns have evolved over the past decades. In the past, while most traffic used to travel up to a Tier 1 provider on its way to its destination, over the years more traffic began to pass over a dense mesh of Tier 2 ISPs rather than transiting major Tier 1 providers. Figure 5(a) illustrates the resulting mesh of Tier 2 peering. The more interesting recent evolution, from the perspective of this paper, is the emergence of large content delivery networks (CDNs), which attach to the various regions of the Internet to facilitate the efficient delivery of content, as illustrated in Figure 5(b). This figure emphasizes the physical topology of interconnection, not distinguishing CDNs from ISPs in terms of their role in the ecosystem.

Illustrating interconnection in terms of layers of platforms, as in Figure 6(a), clarifies this distinction. In this figure, content delivery networks operate at a layer above the global Internet platform, because their role in the ecosystem is not general interconnection among firms that constitute the global Internet, but rather as a service to content providers. A CDN is a complementor of the ISP platforms to which it connects, as well as being another platform in its own right, acting as a delivery enhancement mechanism for a range of higher-level services. Content-related complementors (e.g. a Content Delivery Network or CDN and its customers) are superficially similar to ISPs: they have AS numbers, interconnect with other ISPs, etc. But interconnection between a $\mathrm{CDN}$ and a broadband access provider crosses platform layers, between complementor and Internet. In other words, some physical interconnection involves the construction of the multi-firm platform, and some interconnection represents a use of that platform.

Spulber and Yoo [22] identify five sorts of interconnection that can be found in the Internet: retail (our end-users), wholesale, interconnection (our multi-firm production of the Internet platform), platform (the compementors) and unbundled access (unbundling at the physical layer). They stress the difference between the retail and platform interconnection, although they discuss the platform interconnection mostly in terms of access to TCP/IP, rather than as physical interconnection. They recognize, as we stress, that two sets of links may be identical in terms of physical 


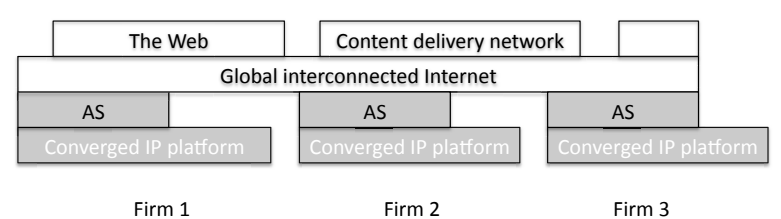

(a) Platform model of the multi-firm Internet, created by interconnecting Autonomous Systems (ASes), i.e., parts of the global Internet operated by one firm.

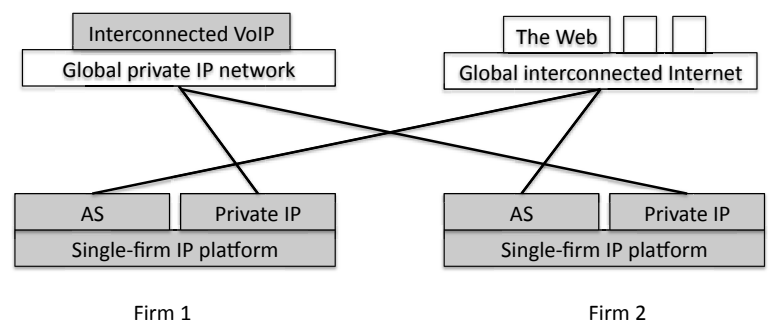

(b) Both the global Internet and a multi-firm internal IP network built by interconnecting the platforms of multiple firms.

Figure 6: The converged IP platform supports two global platforms: (a) the public Internet and $(b)$ a private IP network. Lines in (b) represent physical interconnections as well as connections between platform layers.

properties but different in terms of the economic relationships. The paper presents an economic analysis of the implications of using regulation to manage each of these forms of interconnection.

\subsection{Alternative multi-firm IP platforms}

A more recent development in this evolutionary trajectory is that firms that have built the multifirm global Internet by interconnecting their single firm platforms may replicate this approach to produce other multi-firm IP (but not Internet) platforms. The initial role seen in practice for such a network is as an internal platform, serving the firms that create it. For example, some firms that offer VoIP are now creating a second connected mesh of private IP services over which to interconnect VoIP calls. Figure 6(b) illustrates the creation of both the global Internet (an industry platform) and a multi-firm internal IP platform by the interconnection of single-firm platforms across multiple firms. Just as a single-firm IP platform can serve as an industry platform as well as an internal platform, a multi-firm IP platform could be offered as an industry platform to complementors. Such an interconnected IP platform would be an alternative (to the Internet) industry platform for third-party complementors to reach consumers instead of the current global Internet, perhaps offering better quality of service or security. An infrastructure provider might market such a platform to large application providers such as Facebook as a more effective way to reach consumers, e.g., see figure 7. As with single-firm IP platforms, the inevitable question for the regulator is how to view companies building, interconnecting, and selling services on their own private IP-based platforms, logically if not physically separate from the global Internet platform.

The FCC's Open Internet Report and Order [8] generates some urgency to this question of when an alternative service offering is similar enough to the global Internet that it should be required to comply with the order. The current view expressed (although perhaps not clearly) in this report is that the answer depends on the reach and character of the alternative service: if it reaches essentially as far as the public Internet, then it should comply with the Order, which would impose rules about discrimination and blocking [8, para. 47,112]. However, the multifirm IP platform that carries VoIP services for the facilities owner (e.g., it is currently an internal platform) will likely end up with a global scope, and is not currently subject to neutrality rules. It is not clear why reach is the correct criterion for regulatory classification of a service.

In this section we have mapped various IP-based platforms into our matrix of options for the creation and exploitation of platforms. We have shown examples of an IP platform in each of the four quadrants, and explained how a platform-aware understanding of interconnection can shed 
light on otherwise confusing innovations in the ecosystem. Next we introduce another relevant aspect of platform theory that is essential to understanding the economics of today's Internet infrastructure: the multi-sided platform, or MSP.

\subsection{Multi-sided platforms (MSPs)}

A Multi-Sided Platform (MSP) is a special case of a multi-sided market. A multi-sided market is a circumstance in which a provider has several classes of mutually dependent participants. Hagiu [14] provides a discussion of different definitions of MSPs. He also follows the tradition of using the term customers to describe the classes that are associated with the market. However we will use the term participant to stress the point that the classes of participants that associate with the provider of the market may not all be paying to use the platform. The classic example of a multi-sided market is a dating club, where both men and women must attend for

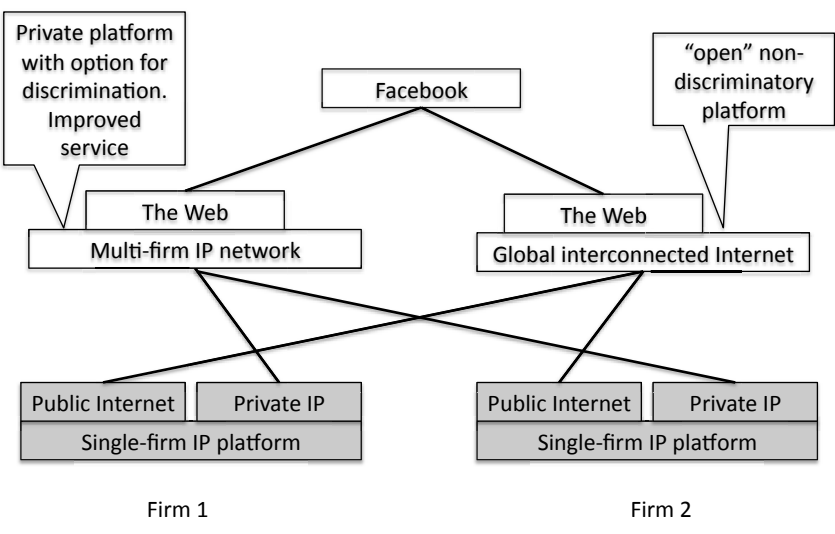

Figure 7: Both the global Internet and a multi-firm IP network acting as alternative industry platforms for consumerfacing services. the club to be successful, and a club owner might offer men and women different prices to entice them both to come, because of a perception that men and women would attach different values to the experience. An MSP, by analogy, is a platform that requires several classes of participants to partake in order for the platform to be successful.

By this definition, the global Internet is an MSP, with retail users as one class of participants and complementors as another. Complementors may participate in a number of ways: providing software, web content, gaming, music sharing, or other services. Complementors and users are mutually dependent; for any activity involving both classes, there is a balance between their roles. Wikipedia is a complementor on the global Internet platform that provides a service (itself a single-firm industry platform) to enable users (contributors) to produce value. For peer-to-peer file sharing, the complementor only produces and perhaps maintains the sharing software, and users create value by using it. For commercial content creation and distribution, the user is a somewhat passive consumer and the complementor drives production.

While the global Internet taken as a whole is arguably an MSP, from both a business and regulatory perspective the unit of concern is usually the firm (or a class of firms, like broadband access providers), not a multi-firm platform. This distinction raises the question of whether it is legitimate to view an individual firm as an MSP. According to Hagiu's definition [14], an MSP's classes of participants must have a direct association with the platform provider. Historically, a complementor attached to the Internet at one or a few points, but served all users on the Internet from those points. Most access ISPs transited packets to and from their peers, but did not have explicit relationships or even necessarily know about any complementors. Even today, most complementors who provide services using network-attached servers (e.g. Web servers or game servers) pay to attach to the Internet, but since much of the Internet interconnects via revenue-neutral peering, no revenues from complementors flow into the access ISP. Retail consumers of broadband access ("eyeballs") cover the entire costs of the access ISP.

This oversimplified description of the Internet would not classify most individual access ISPs as MSPs, since the complementors as a class of participants do not have a direct association with 
the access ISP. There are some exceptions to this case, of course. For example, ISPs that provide Wi-Fi hot spot access for free, financed by advertising revenues, seem like MSPs, with users and advertisers forming the two classes of participants. But with respect to complementors as a class of participants, an explicit association between complementors and access ISPs is increasingly common today, moving individual access ISPs toward an MSP role in the ecosystem. Most notably, large content providers such as Netflix and Google, as well as CDNs that serve content on behalf of many other content providers, often negotiate business relationships that result in direct interconnection to access ISPs, as illustrated in Figure 5(b). As another example of a direct association between access ISP and complementor, HBOgo (the Internet version of HBO) relies on the access ISP to verify that a specific user has the right access credentials based on that customer also subscribing to $\mathrm{HBO}$ via their cable TV service. ESPN requires that access ISPs pay a fee to ESPN in order that its users have access to the Internet ESPN service, called ESPN3. As described in Section 2.2, physical interconnection can reflect either the production of the multi-firm Internet platform or the connection of a complementor to an IP platform; supporting this latter type of interconnection turns an access ISP into an MSP.

\section{Regulatory implications of a multi-sided platform model}

Analyses of multi-sided markets usually focus on differential pricing [17], but much of the concern today with regulation of access ISPs relates to other forms of discriminatory behavior, e.g., blocking or variable quality of delivery of content from complementors. In an analysis of regulatory implications of MSP behavior, Evans [10] concludes that a potential regulator must be cautious about determining predatory pricing in an MSP, as the price on one side may be efficiently set above or below cost, as part of the overall pricing analysis. Generalizing Evans' argument suggests that not just pricing but all forms of potential discrimination should be evaluated with regard to all classes of participants. Several researchers have explicitly argued that certain forms of discrimination can enhance the overall health of an MSP $[9,13,2]$.

For example, the Apple app store (a single-firm industry platform) is an MSP, with two classes of participants: the application designers and the purchasers. Apple discriminates among applications (the complementors). While this business strategy may restrict competition and innovation at the level of the complementors, a thorough MSP analysis should examine the impacts on all platform participants. Discrimination with respect to applications arguably makes the platform more attractive to users, by giving them confidence that undesirable or risky applications have been removed from the system. This outcome might in turn increase market share and make the platform more attractive to complementors. (Note the large difference between an MSP exercising some degree of discrimination with respect to a complementor class and a powerful MSP blocking a popular application, perhaps as an exercise in rent-seeking or protection of a vertically integrated competitor. Apple removed the Google maps app from the iPhone in favor of their own maps, triggering a significant consumer backlash.)

In the remainder of this section we explore how multi-sided platform theory would influence discourse on different types of discrimination by platform owners against complementors and retail users.

\subsection{Discrimination with respect to Internet complementors}

Analysis of the consequences of a particular form of discrimination will depend on the higherlevel goal that drives the call for non-discrimination. Why are advocates and regulators con- 
cerned about non-discrimination on the Internet? One goal is fostering innovation, i.e., nondiscriminatory access protects complementors [1]. Others have argued that non-discriminatory access fosters the production of public and social goods [11]. But as Evans' argument suggests, and Apple's ecosystem demonstrates, some forms of discrimination with respect to the complementors might result in a platform that appeals to more users, and thus fosters more production of downstream goods. The potential complexity of the MSP suggests that protection of complementors and promotion of downstream goods (including public and social goods) merit distinct regulatory analysis with respect to discrimination.

With respect to complementors, ISPs impose some coarse discrimination behavior today, via volume discounts, which favor large, entrenched complementors over new entrants. ISPs can also impose discrimination using deep packet inspection (DPI) to distinguish and prioritize traffic, or limiting a class of application via software on an attached device. ${ }^{4}$ ISPs could also offer discriminatory terms for physical interconnection to complementor networks. The MSP model suggests that all forms of potential discrimination imposed by the platform owner on their complementors should use one regulatory logic, as opposed to viewing physical interconnection through one lens, DPI through another, and so on. The concern is the business relationship, not the technical approach to discrimination.

We are certainly not the first to argue that a multi-sided analysis is appropriate to understand the relationship between complementors and users. Bourreau, Kourandi and Valletti [3] use the concept of the two-sided market to model the relationship between content producers and retail customers. Their framing is similar to ours, in that they are interested not just in pricing, but in the influence of discrimination (or not) among the complementors (in this specific model providers of free content supported by advertising) on consumers, and the resulting implications for the overall ecosystem. They conclude that allowing ISPs to offer enhanced QoS to complementors in exchange for payment is welfare-enhancing. They also conclude from their model that it may be in the interest of an ISP to degrade the basic best-effort service, so that regulatory attention may be required. Their work models two competing ISPs; they cite earlier work that looks at a single monopoly ISP. Sidak [20] has a similar analysis in which he concludes that it is beneficial to allow ISPs to charge content providers for enhanced QoS.

\subsection{Peering disputes and pricing analyses}

In the last five years we have seen increasingly public debates about whether peering between networks should be revenue-neutral (without financial settlements between peers) or whether paid peering should reflect relative costs to ISPs of carrying traffic, or even reflect manifestations of relative bargaining power. The MSP model suggests that regulatory scrutiny of peering disputes should distinguish between two different circumstances: disputes among the firms making up the platform, or discrimination by platform operators on complementors on the platform. A peering dispute between an ISP and a complementor should be subject to the same sort of analysis as any other form of discrimination imposed by an ISP on a complementor.

With respect to peering disputes among firms that interconnect to make up the Internet, such events are characteristic of what can happen in any multi-firm platform. As described by Gawer [12], in any multi-firm platform a powerful actor can dominate the creation of the platform, or potentially even disrupt it by predatory behavior. In particular, the argument is much weaker that an ISP is situated in an MSP when it negotiates terms for peering with other ISPs than when it negotiates with complementors who need access to users.

\footnotetext{
${ }^{4}$ For example, ATT used a feature in the iPhone software to block use of the FaceTime app for certain data plans. See http:/ / arstechnica.com/apple/2013/01/att-continues-chipping-at-facetime-over-cellular-policy/.
} 
Peering disputes with complementors have gained more recent attention, given the increasing number and size of complementors seeking direct associations with access ISPs. Some access ISPs have questioned the proper balance of pricing and discrimination between a specific class of complementor (content distribution networks) and users, arguing that distributors of content should pay for the right to connect to the ISP's customers [18]. This situation is a classic example of a pricing analysis in an MSP; previously, one class of participant, the end-consumer, was covering the costs of the access ISP; an alternative is to extract some of that revenue from another class of participant. Although this move might signal the exercise of market power by the ISP, Evans argues [10] that the presence of market power does not affect the analysis of efficient pricing in an MSP. Extraction of rents is an issue with market power, efficient pricing is not. So, ignoring the issue of market power, what are the implications of shifting the balance of payments between users and complementors of the access ISP? It is unreasonable to assume that the ISP could exercise precise first-degree price discrimination and charge each complementor a price that reflects their willingness to pay, given both its impracticality as well as the likely reaction from the market and the regulators. A more likely approach is for the access ISP to charge complementors per bit of data generated by the service, which might drive out of the market complementors with low or unproven value. On the other hand, if this shift significantly drove down the price charged to users, more users might enter the market.

We know of no fundamental principle that resolves this balance; it calls for empirical data such as cost and price elasticity. However, the complexity of the MSP landscape implies that ISPs contemplating a shift in their negotiating position about interconnection with complementors should not consider the shift only as a new source of revenues. Even non-disciminatory prices charged to complementors, if high enough, could chill innovation. Lower prices may benefit innovation and public good creation more than the total absence of discrimination, especially in the complementor market.

\subsection{Discrimination with respect to retail users}

As a preliminary to the rules promulgated in the Open Internet Report and Order, and to frame their regulatory objectives to "ensure that broadband networks are widely deployed, open, affordable, and accessible to all consumers", the FCC published four principles [6]:

To encourage broadband deployment and preserve and promote the open and interconnected nature of the public Internet, consumers are entitled to

- access the lawful Internet content of their choice.

- run applications and use services of their choice, subject to the needs of law enforcement.

- connect their choice of legal devices that do not harm the network.

- competition among network providers, application and service providers, and content providers.

The first two principles, without using the language or framing of an MSP, in fact do a twosided analysis, looking at the consequences for the consumer (one class of participant) of discrimination on another class (complementors-providers of content and applications). The third principle directly protects the user from one form of discrimination, and the fourth principles offers a bit of wishful hoping that competition happen. But the only direct protection of the user from discrimination is that the consumers can attach devices of their choice. There is no requirement that all potential users be served, or served at the same price. 
In fact, ISPs today do discriminate with respect to what consumers can do. One common limitation is that residential users are not allowed to attach a "server" (device) to their network. This restriction might fall within the "reasonable network management" exception on a cable system with limited upstream capacity, but when Google imposed the same restriction on their Kansas City service with abundant upstream capacity, they received some criticism [21]. Another view is that the the limited protection given to the consumer by the four principles was intentionally constructed to allow ISPs to stratify their users into classes, e.g., residential vs. business.

With respect to pricing, most wireline ISPs do not differentially charge users based on what they are doing. Rather, they offer either flat pricing or usage caps. These billing models are a form of discrimination: some customers with low usage support those with higher usage. The benefit to this form of discrimination is that for any individual user, the incremental cost of trying some new service from a complementor, or engaging in an activity that generates mostly a public good (e.g. the user does not appropriate the benefit) is zero. In contrast, wireless plans that impose usage caps and tiers may inhibit experimentation and participation in activities where the value that accrues to the user is (perceived to be) low.

\subsection{MSP behavior in more mature markets}

Another consequence of applying MSP theory relates to saturation in one side of the market. According to MSP theory, when platforms compete (as with IP vs. another protocol suite) in a growing market, innovation by complementors brings value to the platform owner in the form of network effects that lead to success of the platform. This dynamic applied in the early days of the Internet. But once the platform (as an interface or standard) has achieved market dominance, and especially if the pool of users saturates, as it seems to be doing for land-line broadband access, the logic of the MSP may cease to explain the benefit to the platform provider of more complementors (i.e., apps). Indeed, with current wireline pricing models that bring little or no additional revenues with additional usage, new complementors that drive increased usage bring negative value to the platform provider. Alternatively, usage tiers may encourage investment in capacity, and thus the addition of new complementors, but at the same time discourage the use of these complements by cost-conscious users.

\section{Shaping consistent regulation based on layered MSP model}

Our repeating layer model captures the idea that a given actor (e.g., an ISP) may produce many platforms as part of their system design. We have focused on three platforms that appear (or are likely) to be stable in the ecosystem: the global Internet, the single-firm IP platform, and a multi-firm IP platform. We have also explored how the theory of multi-sided platforms can shed further light on the behavioral influences of and on platform owners, complementors, and users. Since regulation attached to stable layers would likely be more durable, we now consider how the framework of platforms we have developed can help scope consistent policy and regulation of the Internet. We examine three open issues and what light platform theory sheds on them: one shortterm regulatory quagmire, one medium-term policy challenge, and one long-term philosophical debate.

\subsection{Consistent regulation of services on different IP-based platforms}

Regulators, when looking at the structure of the market, may look at the overall behavior of firms, or at the desired qualities of a platform. Put differently, when should a regulator consider a plat- 
form in isolation, as opposed to considering a firm's treatment of several platforms. MSP theory suggests an answer to this question: platform layers merit a unified analysis if they serve the same classes of participants. Whether the platform is single-sided and serves one class of participants, or an MSP serves multiple classes, if two platforms can serve the same classes then they should be considered jointly. As a specific example, if an access ISP offers its single-firm IP platform as an alternative industry platform for (at least some) complementors to reach consumers, the single-firm and global Internet platforms are alternative means to serve the same classes of participants, and merit joint consideration. In contrast, an access ISP might use its single-firm MPLS platform layer to offer an "Ethernet-over-MPLS" service to allow a multi-site enterprise to connect its various sites; this type of enterprise constitutes a different class of customer, and thus this platform merits independent consideration from an MSP IP platform.

Note that joint consideration of platforms does not imply equal treatment. The single-firm IP platform as a industry platform for complementors imposes extra burdens on potential complementors: it serves only complementors that arrange to connect directly to it. If (as we described earlier) multiple access ISPs interconnected their single-firm IP platforms to build a second, multifirm IP industry platform that served the same set of user classes (complementors and consumers) with the same implications for those classes of participants, it would seem the two platforms would be more likely to deserve similar regulatory treatment. Some entanglement potentially arises between platforms if they serve some but not all of the same participant classes. As in the Xfinity Xbox service (Section 2.1), an access ISP could use its internal single-firm IP platform to offer a video service to consumers (e.g., it could act as a reseller of video content over that platform), which arguably competes with a video service offered by a third-party complementor over the global IP platform. The services over the two platforms (single-firm IP and the Internet) have the retail user in common as a class of participants, but they do not involve the same classes of participants: with carrier IPTV, the ISP is acting as a reseller of video programming, not as an MSP. Any entanglement of regulatory consideration would derive from the fact that services over both platforms offer competing and similar offerings to one class of participants.

We observe that the FCC tried to address this situation by using the term "specialized services" in the Open Internet Report \& Order [8]; a specialized service is any service that is not burdened with the obligations of the report and order. Like many other terms introduced to describe new behaviors that do not fit into the prevailing model of industry behavior, the term "specialized services" brings little clarity to what is happening. The FCC specifically identifies carrier IPTV as a specialized service (e.g., IPTV running over the single-firm IP platform of the provider, although they do not use this terminology or framing), but the more general (and not yet resolved) question is when to classify a service as a specialized service. The FCC's current position is that the distinction is determined by the set of machines reachable via the service [8, para. 47]. Comcast, in describing their Xfinity IPTV service, stressed that the IPTV and Internet service use different logical slices of Comcast's bandwidth capacity. ${ }^{5}$ These sorts of distinctions do not seem useful as a test of whether a service is running on the internal IP platform or on the global Internet service. MSP theory suggests that a more fundamental and promising starting point for classification of specialized services is the degree to which the two services have common classes of participants. Thus, carrier-provided IPTV is a video reseller service that competes with services that complementors offer over the global Internet. In an MSP-based framework, the basic question is whether this type of competition between platforms should warrant any regulatory attention, not the details of how capacity is allocated or routing is restricted.

\footnotetext{
${ }^{5}$ See http://corporate.comcast.com/comcast-voices/the-facts-about-xfinity-tv-and-xbox-360-comcast-is-notprioritizing
} 


\subsection{Regulating the platform itself rather than the platform provider}

The previous section described an approach to regulation in which the regulator imposes requirements on a firm, perhaps across several platforms, to limit its behavior with respect to various classes of its users, such as complementors. An alternative is to regulate a specific platform, with the goal of sufficiently satisfying high-level social goals (e.g., high performance, low cost, complementor innovation) so that regulation of other platforms of the firm is not required. Such platform regulation will attempt to ensure that the player with power (the platform provider) is working for the overall good of the ecosystem, but this abstract policy statement leaves much unanswered about the right approach. We examine potential regulation of the global Internet platform as a specific case study.

The higher-level question remains: what goal might warrant regulatory intervention? If the goal is to drive healthy innovation, then if the global Internet is "good enough" to stimulate complementor innovation, it matters less what the provider does with its single-firm IP platform. In other words, the higher performing and lower cost the global Internet offering, the lower the regulatory interest should be in activities on the single-firm (or multi-firm) IP layer. (This approach raises the question of how to define the minimum quality standards, which others have already recognized as a potential anchor of future regulation $[4,5]$.)

The traditional discourse on network neutrality (and specifically in the U.S., the FCC Open Internet principles [6]) approaches this question primarily by constraints on the Internet offering itself. But the alternative platforms, particularly the single and multi-firm IP platforms, increase the degrees of freedom both for an ISP and for the regulator. Any theory of regulation that argues for limitation on the activities of the facilities owner must take into account the degrees of freedom that the platform owner has. For example, imagine that a platform operator (broadband provider) allocates a share of the IP platform as an alternate multi-firm industry platform, which is IP-based, but not interconnected with the public Internet. On that share, the platform operator allows thirdparty complementors to offer consumer-facing services, perhaps with superior qualities compared to the public Internet (e.g. QoS), or may curate the available applications like the Apple app store. A theory of regulation should be able to explain why this business behavior is pro-innovation or anti-competitive, pro-consumer or not. The MSP analysis can illuminate debate of these sorts of questions. It may reveal, given the degrees of freedom to both actors, that a better regulatory approach is to offer incentives to the ISP to improve the global Internet platform, as we describe above, rather than constraining its operation.

\subsection{Structural separation}

Finally, we use our multi-sided platform model to describe and compare options for a more radical approach to industry regulation. In platform terms, structural separation is a requirement that the owner of some platform be required to use it only for third- party complementors, but not as an internal platform for deploying higher-level services. Structural separation is a extreme form of facilities unbundling; in simple unbundling, the owner of the platform must make it available to any comer on non-discriminatory terms, but is also allowed to use it as an internal platform on the same terms.

As a real-world example, the British regulator has imposed structural separation on the physical layer platform of British Telecom (BT). BT was required to establish a separate firm, OpenReach, to own and operate the actual copper pairs. In this paper we have not emphasized the platform represented by the physical layer, but it is indeed another durable layer and thus a reasonable target of regulation. However, several aspects of the physical layer make it a challenging 
target of structural separation regulation. First, this layer is very dependent on particular technology choices. It is difficult to imagine a technology-neutral form of structural separation at the physical layer. Second, using copper pairs as an example, there is no simple way to share a copper pair between two complementors. A given consumer will pick among competing providers of higher-level services, but then must obtain all services from that one provider. The resulting market structure would seem to limit competition among complementors.

Imposing a separation point at a higher-level platform would allow more flexibility in the resulting market structure. In particular, separation at a layer that allows multiplexing among multiple complementors (e.g., a packet-switched platform) could allow multiple complementors to offer services to the same customer. The packet level could be the single-firm IP platform, or a lower-level platform such as an ATM platform. Our emphasis on finding durable platform layers around which to regulate implies that structural separation of an intermediate layer (between the physical and the single-firm IP platform) would be most likely to succeed if the platform where separation was imposed was defined in a technology-independent way, and thus could apply to a range of alternative technologies that will be found in those layers over time.

We are not advocating structural separation; just framing it in terms of our model. But the tools of MSP and multi-platform analysis could help to answer the question in any debate over structural separation: what are the implications for the platform owner and the larger ecosystem if the owner were precluded from using it as an internal platform.

\section{Conclusions}

We have presented a model that characterizes both the layered platform architecture of today's communications technology, and aspects of interconnection among firms to produce what we have identified as the global Internet and its connected complementors. We have identified three specific IP-based platforms as important: the single-firm IP platform, the global Internet, and a multi-firm private IP platform. These platform layers will be more stable fixtures of the infrastructure than other layers beneath or above them, and thus a focus on these specific layers will help produce policy that is durable in the face of evolving business and technology. Distinguishing the three is necessary in any realistic regulatory or business analysis. With respect to issues related to interconnection, we argue that distinguishing between the role of multi-firm production and complementor interconnection is a fundamental criterion. Peering among firms that make up the global Internet raises different issues than interconnection between a complementor and the Internet.

Our model suggests opportunities for discrimination on multi-sided platforms, both with respect to users and to complementors. These include pricing discrimination (some of which we see today, such as volume discounts and flat rate pricing), discrimination in interconnection policy, and manipulation of traffic flows based on their characteristics, including which users or which complementors generate the traffic. In trying to understand which of these behaviors should be treated together rather than separately, we argue that with respect to a particular platform, all forms of discrimination with respect to a given class of participant (e.g., a complementor) should be analyzed using the same framework, as opposed to looking at (for example) interconnection using one framework and deep packet inspection using another. With respect to the question of whether behavior on different platforms should be evaluated independently or in common, we argue that a starting point is to determine the extent the two platforms serve the same set of participants. 


\section{References}

[1] Barbara van Schewick. Internet Architecture and Innovation. MIT Press, 2010.

[2] Kevin Boudreau and Andrei Hagiu. Platform rules: Multi-sided platforms as regulators. In Platforms, Markets and Innovation. Edward Elgar, 2011.

[3] Marc Bourreau, Frago Kourandi, and Tommaso Valletti. Net neutrality with competing internet platforms, 2012. Available at SSRN: http: / / s srn . com/abstract=2061571 orhttp: //dx.doi.org/10.2139/ssrn.2061571.

[4] Tim Brennan. Net Neutrality or Minimum Quality Standards: Network Effects vs. Market Power Justifications, 2010. https://papers.ssrn.com/sol3/papers.cfm? abstract_id=1622226.

[5] kc claffy and David. Clark. Workshop on Internet Economics (WIE2012) Report. ACM SIGCOMM Computer Communication Review (CCR), July 2013.

[6] Federal Communications Commission. Fcc 05-151, policy statement, 2005. In the matter of Appropriate Framework for Broadband Access to the Internet over Wireline Facilities, CC Docket No. 02-33, etc., http://hraunfoss.fcc.gov/edocs_public/attachmatch/ FCC-05-151A1.doc.

[7] Federal Communications Commission. In the matter of implementation of section 621(a)(1) of the cable communications policy act of 1984 as amended by the cable television consumer protection and competition act of 1992, 2005. MB Docket No. 05-311, FCC 06-180.

[8] Federal Communications Commission. Report and order, preserving the open internet, fcc10-201, 2010. http://hraunfoss.fcc.gov/edocs_public/attachmatch/ FCC-10-201A1_RCd.pdf.

[9] Thomas Eisenmann, Geoffrey Parker, and Marshall Van Alstyne. Open platforms: How, when and why. In Platforms, Markets and Innovation. Edward Elgar, 2011.

[10] David A. Evans. The antitrust economics of multi-sided platform markets. Yale Journal on Regulation, 20(2), 2003.

[11] Brett M. Frischmann. Infrastructure: The Social Value of Shared Resources. Oxford University Press, 2012.

[12] Annabelle Gawer. Platform dynamics and strategies: From products to services. In Platforms, Markets and Innovation. Edward Elgar, 2011.

[13] Shane Greenstein. Open platform development and the commercial internet. In Platforms, Markets and Innovation. Edward Elgar, 2011.

[14] Andrei Hagiu and Julian Wright. Multi-sided platforms. Technical Report 12-024, Harvard Business School, 2011. available at http: / hbswk . hbs . edu/item/6681.html?

[15] Scott Jordan. A layered network approach to net neutrality. International Journal of Communication, 1:427-460, 2007.

[16] Rob Frieden. The mixed blessing of a deregulatory endpoint for the public switched telephone network. Telecommunications Policy, Iuly 2012. 
[17] Jean-Charles Rochet and Jean Tirole. Two-sided markets: A progress report. The RAND Journal of Economics, 37(3):645-667, 2006. http: / /www . jstor. org/stable/25046265.

[18] Sean Buckley. Cogent and Orange France fight over interconnection issues. Fierce Telecom, 2011.

[19] Douglas Sicker and Joshua Mindel. Refinements of a layered model for telecommunications policy. Journal on Telecommunications and High Technology Law, pages 69-94, 2002.

[20] J. Gregory Sidak. What is the network neutrality debate really about? International Journal of Communication, 1, 2007. http://ijoc.org/index.php/ijoc/article/view/177/95.

[21] Ryan Singel. Now that it's in the broadband game, google flip-flops on network neutrality, 2013. http://www.wired.com/threatlevel/2013/07/google-neutrality/.

[22] Daniel F. Spulber and Christopher S. Yoo. Network regulation: The many faces of access. Journal of Competition Law and Economics, 1(4):635-678, 2005.

[23] Philip J. Weiser. Law and information platforms. Journal on Telecommunications and High Technology Law, pages 1-36, 2002.

[24] Kevin Werbach. A layered model for internet policy. Journal on Telecommunications and High Technology Law, pages 37-68, 2002. 